\title{
Constitutive Expression of VvAATP Increases Starch Content in Transgenic Arabidopsis
}

\author{
Feibing Wang ${ }^{1, *}{ }^{*}$, Bin Weng ${ }^{1, \dagger}$, Qi Wang ${ }^{1}$, Yuxiu Ye ${ }^{1}$, Jing Zhang ${ }^{2}$, Gaolei Ren ${ }^{1}$, Bowen Wang ${ }^{1}$, \\ Qing Zhou ${ }^{1}$, Xinhong Chen ${ }^{1, *}$
}

${ }^{1}$ School of Life Science and Food Engineering, Huaiyin Institute of Technology, Huai'an, China

${ }^{2}$ School of Chemical Engineering, Huaiyin Institute of Technology, Huai'an, China

Email address:

wangfeibing1986@163.com (Feibing Wang),cxh_xj@163.com (Xinhong Chen)

${ }^{*}$ Corresponding author

$\dagger$ Feibing Wang and Bin Weng have contributed equally to this work

\section{To cite this article:}

Feibing Wang, Bin Weng, Qi Wang, Yuxiu Ye, Jing Zhang, Gaolei Ren, Bowen Wang, Qing Zhou, Xinhong Chen. Constitutive Expression of VvAATP Increases Starch Content in Transgenic Arabidopsis. Biochemistry and Molecular Biology. Vol. 2, No. 6, 2017, pp. 94-101.

doi: 10.11648/j.bmb.20170206.15

Received: September 5, 2017; Accepted: October 18, 2017; Published: November 22, 2017

\begin{abstract}
A plastidic ATP/ADP transporter (AATP) plays a crucial role in importing ATP from the cytosol into plastids, leading to the increase the ATP supply to facilitate anabolic synthesis in heterotrophic plastids of dicotyledonous plants. The regulatory role of the grapevine $V v A A T P$ gene in increasing starch accumulation has not been investigated. In this study, the VvAATP gene was successfully isolated from grapevine and transformed into Arabidopsis. Constitutive expression of VvAATP significantly increased starch accumulation in transgenic Arabidopsis plants. Real-time quantitative PCR analysis showed that constitutive expression of $V v A A T P$ up-regulated the expression of the genes related to starch biosynthesis pathway, including phosphoglucomutase, ADP-glucose pyrophosphorylase (AGPase), granule-bound starch synthase (GBSS), soluble starch synthase (SSS) and starch branching enzyme (SBE) genes, in transgenic Arabidopsis plants. Meanwhile, enzymatic analyses indicated that the major enzymes (AGPase, GBSS, SSS and SBE) involved in the starch biosynthesis exhibited higher activities in the transgenic plants compared to the wild-type (WT). These results indicate that $V v A A T P$ may improve starch content of Arabidopsis by up-regulating the expression of the related genes and increasing the activities of the major enzymes invovled in starch biosynthesis. All these findings suggest that the $V v A A T P$ gene may be applied for increasing starch accumulation of plants in the future.
\end{abstract}

Keywords: Arabidopsis, Constitutive Expression, Grapevine, Starch Content, VvAATP

\section{Introduction}

Biofuel, which can decrease environmental damage by reducing the extraction and use of fossil fuels, is more and more important with the society's development. Breeding of non-food energy crops for economically viable production as environmentally friendly biofuels is on the way [1]. Due to the conversion of starch into fermentable sugars is relatively easy, starch has been taken as a major feedstock for first-generation biofuel production $[1,2]$. Therefore, it is vital to know how carbohydrates are metabolized in plants, which could be of great help in the development of crops by means of enhancing starch synthesis, and in the improvement of biofuel production efficiency [1].

As a main source of nutrition in the human and animal diet, starch is a major storage of carbohydrates in plants [3], and plays a fundamental role in plant survival and adaptation to various environmental conditions [4]. In plants, starch exists as an insoluble glucan, comprised of two glucose polymers: amylose and amylopectin. Amylose is mainly comprised of linear chains that are linked by $\alpha-1,4$ O-glycosidic bonds, whereas amylopectin is highly branched and contains 5\%-6\% $\alpha-1,6$ O-glycosidic bonds to generate glucan branches of various lengths [5]. Four major enzymes are involved in starch biosynthesis: ADP-glucose pyrophosphorylase (AGPase), starch synthase (SS), starch branching enzyme (SBE) and 
starch debranching enzyme (DBE) [6].

In below-ground storage organs, the best option to increase the starch content may lie in increasing the ATP supply to the plastid [2]. ATP is the basic energy currency in living cells and is needed for almost every step of biochemical reactions. ATP is also an indispensable participant in the AGPase reaction, which catalyzes the formation of ADP-glucose (ADPG) and is considered as a rate-limiting enzyme in starch biosynthesis [7] ATP exchange between organelles and the cytosol is mediated by adenylate carrier proteins. One type of adenylate carrier protein is the mitochondrial ADP/ATP carrier (AAC), which can export ATP produced previously via oxidative phosphorylation in a one to one exchange of cytosolic ADP [8]. The mitochondrial AAC is a dimer, and each monomer contains 6 transmembrane helices [9]. Another type of adenylate carrier protein is the plastidic ATP/ADP transporter protein (AATP), which was discovered in spinach chloroplasts. AATP is generally found in the heterotrophic plastids (amyloplasts, chromoplasts and leucoplasts) of higher and lower land plants as an important energy transporter [10-16]. The main function of AATP is involved in providing the plastid stroma with cytosolic ATP, to further participate in anabolic processes such as starch and fatty acid synthesis [13].

There are a few reports about the function of AATP in starch biosynthesis in plants. Constitutive expression of the Arabidopsis AtAATP1 increased ADP-glucose level up to 2 -fold and starch content by 16-36\% [17]. Down-regulation of the potato StAATP decreased ADPG level by $25-70 \%$ and starch content by $19-51 \%$ in potato tubers [18]. Ectopic expression of SIAATP/StAATP/GmAATP from tomato/potato/soybean increased starch accumulation in the leaves of transgenic Arabidopsis plants [19-21]. Overexpression of IbAATP increased starch and amylose content in transgenic sweetpotato [22]. These results indicate that the manipulation of the enzymes that modulate the ATP supply in plastids is an effective way to enhance starch biosynthesis in plants. However, the regulatory role of the grapevine $V v A A T P$ gene in increasing starch accumulation has not been investigated.

In this study, the $V v A A T P$ (Genbank accession No. Y10821) gene was isolated from grapevine and introduced into Arabidopsis to estimate its roles. Ectopic expression of $V v A A T P$ significantly increased the starch accumulation in the leaves of transgenic plants, indicating a great potential of $V v A A T P$ in the development of high starch-accumulating plants.

\section{Materials and Methods}

\subsection{Plant Materials}

Grapevine cultivar PN40024 was employed for VvAATP gene cloning in this study. Arabidopsis [ecotype Columbia-0, wild type (WT)] was used as a model plant to investigate the functions of $V v A A T P$.

\subsection{Cloning of the Grapevine VvAATP Gene}

Total RNA was extracted from freshly leaves of PN40024 with the RNAprep Pure Kit (Tiangen Biotech, Beijing, China). RNA samples were reverse-transcribed according to the instructions of Quantscript Reverse Transcriptase Kit (Tiangen Biotech, Beijing, China). Based on the sequence of VvAATP (Genbank accession No. XP_002285232), we designed one gene-specific primers $(\mathrm{GC}-\mathrm{F} / \mathrm{R})$ of reverse transcription PCR (RT-PCR) (Table 1) to obtain its full-length cDNA sequence. PCR was performed with an initial denaturation $94^{\circ} \mathrm{C}$ for $3 \mathrm{~min}$, followed by 35 cycles of $94^{\circ} \mathrm{C}$ for $30 \mathrm{~s}, 55^{\circ} \mathrm{C}$ for $30 \mathrm{~s}, 72^{\circ} \mathrm{C}$ for $1 \mathrm{~min}$ and final extension $72^{\circ} \mathrm{C}$ for $10 \mathrm{~min}$. PCR products were separated on a $1.0 \%(\mathrm{w} / \mathrm{v})$ agarose gel. Target DNA bands were recovered by gel extraction, then cloned into PMD19-T (TaKaRa, Beijing, China), and finally transformed into competent cells of Escherichia coli strain DH5 $\alpha$. White colonies were checked by PCR and the positive colonies were sequenced (Invitrogen, Beijing, China). The genomic sequence of $V v A A T P$ was amplified with primers GA-F/R (Table 1) using genomic DNA extracted from the leaves of PN40024 as a template.

\subsection{Sequence Analysis of the VvAATP Gene}

The open-reading frame (ORF) of the cloned $V v A A T P$ gene was predicted with ORF Finder (http://www.ncbi.nlm.nih.gov/projects/gorf/). The full-length cDNA of $V v A A T P$ was analyzed by BLAST on the National Center for Biotechnology Information (NCBI) website (http://www.ncbi.nlm.nih.gov/). For the multiple sequence alignment analysis, the amino acid sequence of $V v A A T P$ and Arabidopsis AtAATP homologs retrieved from NCBI were aligned using the DNAMAN software (Lynnon Biosoft, Quebec, Canada). The theoretical molecular weight and isoelectronic point $(p \mathrm{I})$ were calculated using ProtParam tool (http://web.expasy.org/protparam/). The conserved domain of the VvAATP protein was scanned by the InterProScan program (http://www.ebi.ac.uk/Tools/pfa/iprscan/).

\subsection{Construction of Transgenic Arabidopsis with VvAATP Gene}

The binary vector pCAMBIA1301-VvAATP used in this study contained the $V v A A T P$ gene under the control of the cauliflower mosaic virus (CaMV) $35 \mathrm{~S}$ promoter and the nopaline synthase (NOS) terminator and $\beta$-glucuronidase ( gusA) and hygromycin resistance ( $h p t$ II ) genes driven by a CaMV 35S promoter, respectively. The primers used to amplify $V v A A T P$ were designed with the Primer5 (Table 1). Both pCAMBIA1301-VvAATP and the control vector (VC) pCAMBIA1301 were transformed into the Agrobacterium tumefaciens strain LBA4404 cells by the electroporation method for Arabidopsis transformation [23]. Transgenic plants were produced according to methods described previously [24]. Transformants were selected based on their resistance to hygromycin (Hyg). Putative transformant seeds were germinated on agar-solidified MS [25] medium containing $25 \mathrm{mg} / \mathrm{L} \mathrm{Hyg}$. Positive transgenic seedlings were grown in pots containing a mixture of soil, vermiculite and humus $(1: 1: 1, v / v / v)$ for $T_{2}$ and $T_{3}$ seed selection. The 
incubation and growth conditions of Arabidopsis were the same as described previously [24].

\subsection{Molecular Confirmation of Transgenic Plants}

The presence of $V v A A T P$ in hygromycin-resistant plants was assessed by PCR analysis using specific primers (Table 1) to amplify fragments of the $h p t$ II coding sequence. DNA was first extracted from Arabidopsis leaves according to the instructions of EasyPure Plant Genomic DNA Kit (Transgen, Beijing, China). PCR amplifications were performed with an initial denaturation $94^{\circ} \mathrm{C}$ for $3 \mathrm{~min}$, followed by 35 cycles of $94^{\circ} \mathrm{C}$ for $30 \mathrm{~s}, 55^{\circ} \mathrm{C}$ for $30 \mathrm{~s}, 72^{\circ} \mathrm{C}$ for $1 \mathrm{~min}$ and final extension $72^{\circ} \mathrm{C}$ for $10 \mathrm{~min}$. PCR products were separated by electrophoresis on a $1.0 \%(\mathrm{w} / \mathrm{v})$ agarose gel.

\subsection{Assay for Starch Content}

Starch extraction and quantification were performed as described previously [26]. Seeds were grown on MS medium for 2 weeks and transferred to pots containing a mixture of soil, vermiculite and humus $(1: 1: 1, \mathrm{v} / \mathrm{v} / \mathrm{v})$. Plants were grown in growth chamber for 4 weeks at $22^{\circ} \mathrm{C}$ under standard long day conditions (14 h light and $10 \mathrm{~h}$ dark). Leaves of four-week-old plants were harvested to determine starch content in light at 10-11 a.m. All treatments were performed in triplicate.

\subsection{Expression Analysis of the Related Genes}

The expression of $V v A A T P$ and starch biosynthesis related genes was analyzed by real-time quantitative PCR (qRT-PCR) as described by Wang et al. [19]. The cDNA solution was used as templates for PCR amplification with gene specific primers (Table 1). Arabidopsis Atactin gene (Genbank accession No. NM112764) was used as an internal control [27] (Table 1). Quantification of gene expression was done with comparative $C_{\mathrm{T}}$ method [28]. All experiments were repeated three times and each data represents the average of three experiments.

\subsection{AGPase, GBSS, SSS and SBE Activity Analyses}

The activity of four starch biosynthetic enzymes (AGPase, GBSS, SSS and SBE) in the leaves of four-week-old transgenic, VC and WT plants was performed according to the method described by Nakamura et al. [29]. One unit of enzyme activity (AGPase, GBSS and SSS) was defined as the formation of 1 nmol ADP per min at $30^{\circ} \mathrm{C}$ and 1 unit of SBE activity was defined as the amount of enzyme required to increase the spectrophotometric absorbance by 1 unit in 1 minute.

\subsection{Statistical Analyses}

All experiments were repeated three times and the data presented as the mean \pm standard error (SE). Where applicable, data were analyzed by Student's $t$-test in a two-tailed analysis. Values of $P<0.05$ or $<0.01$ was considered to be statistically significant difference.

\section{Results}

\subsection{Cloning and Sequence Analyses of VvAATP}

The $V v A A T P$ gene was cloned by RT-PCR. $V v A A T P$ contained a 1866 bp ORF, encoding a polypeptide of 621 amino acids, with a molecular weight of $68.12 \mathrm{kDa}$ and a theoretical isoelectric point $(p \mathrm{I})$ of 9.53. Sequence analysis via the Inter Pro Scan program (http://www.ebi.ac.uk/Tools/pfa/iprscan/) showed that the VvAATP protein contained an ADP/ATP carrier protein domain (Figure 1A). The genomic sequence of $V v A A T P$ was 3175 bp long and contained 5 exons and 4 introns. A BLASTP search indicated that the amino acid sequence of $V v A A T P$ shared $76.50 \%$ and $78.08 \%$ amino acid identity to AtAATP2 (Arabidopsis thaliana, NP_173003) and AtAATP1 (A. thaliana, NP_178146), respectively (Figure 1B).

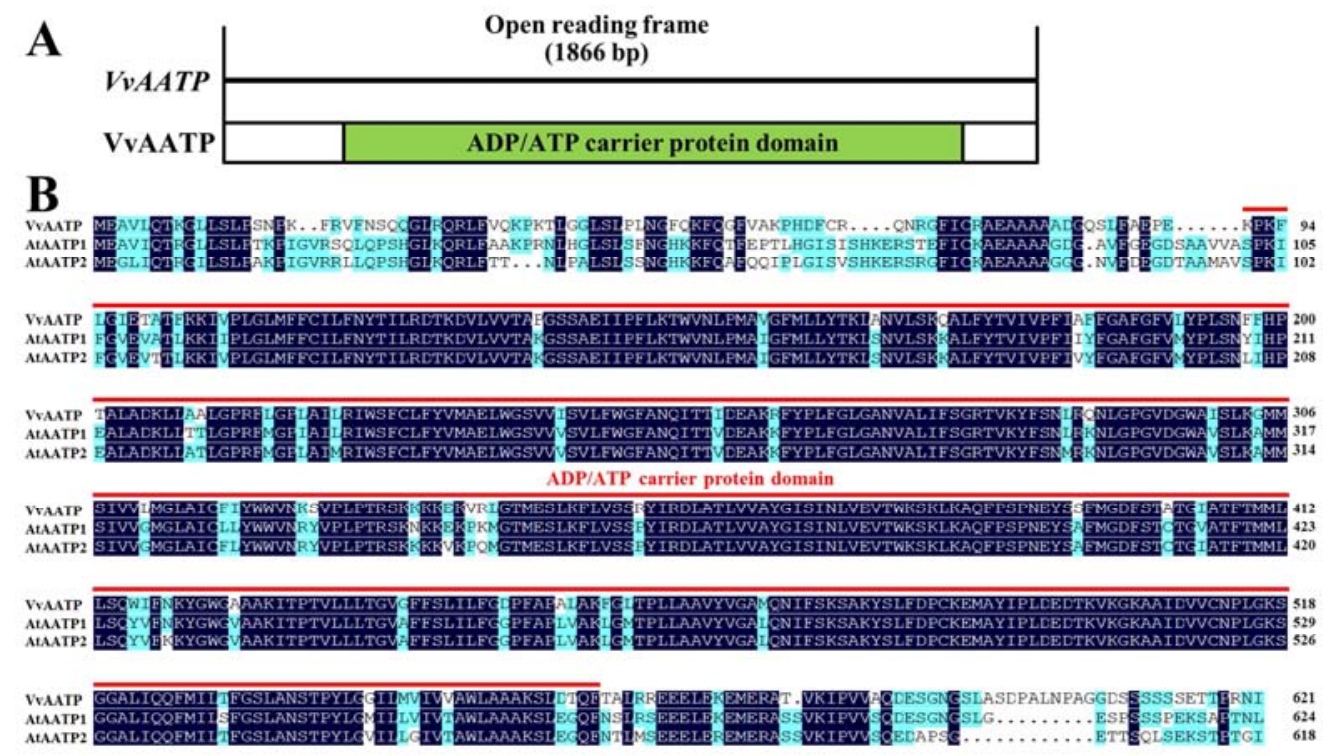

Figure 1. Analyses of the VvAATP gene from grapevine. A, Structure analysis of the VvAATP protein. The VvAATP protein contains an ADP/ATP carrier protein domain. B, Amino acid sequence alignment of VvAATP with Arabidopsis AtAATP1 and AtAATP2. The dark blue in the proteins alignment has higher amino acid sequence alignment, while the light blue has lower amino acid sequence alignment. 


\subsection{Increased Starch Content in Arabidopsis Expressing VvAATP}

The ORF of $V v A A T P$ was ectopically expressed in Arabidopsis (Col-0, WT) using the binary vector pCAMBIA1301-VvAATP (Figure 2A). Six independent transgenic lines constituviely expressing $\operatorname{VvAATP}\left(\mathrm{T}_{1}\right.$ generation) were obtained by Hyg resistance selection, named $\# 1-\# 6$, respectively, and their progenies ( $T_{3}$ generation) were generated. PCR analyses of genomic DNA confirmed the successful integration of transgene (Figure 2B). qRT-PCR analyses showed that the higher expression levels of $V v A A T P$ were observed in transgenic lines \#3 and \#6, while no transgene expression was observed in VC and WT (Figure 2C).

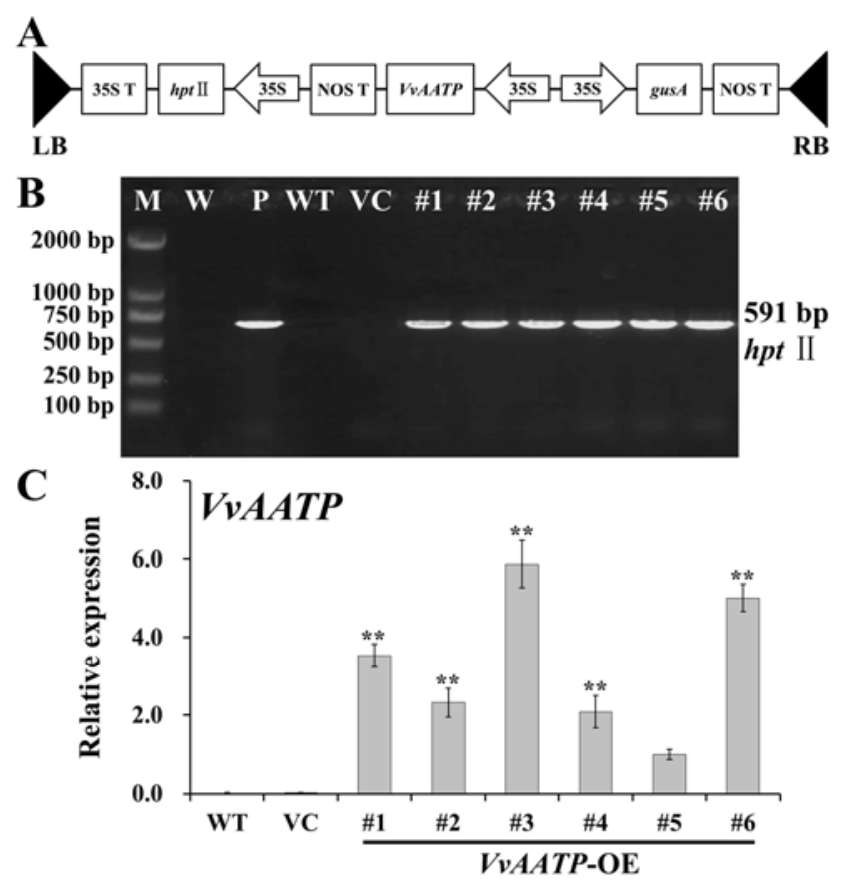

Figure 2. Identification of transgenic plants. A, Schematic diagram of the T-DNA region of binary plasmid pCAMBIA1301-VvAATP. LB, left border; $R B$, right border; hpt II, hygromycin phosphotransferase II gene; VVAATP, grapevine plastidic ATP/ADP transporter protein gene; gusA, $\beta$-glucuronidase gene; $35 S$, cauliflower mosaic virus (CaMV) $35 S$ promoter; $35 S$ T, CaMV 35S terminator; NOS T, nopaline synthase terminator. B, PCR analysis of VVAATP expressing Arabidopsis plants. Lane M: DL2000 DNA marker; Lane $W$ : water as negative control; Lane $P$ : plasmid pCAMBIA1301-VvAATP as positive control; Lane WT: wild type; VC, control vector; Lanes \#1-\#6: different transgenic lines. C, Expression levels of VvAATP in different transgenic lines. The Arabidopsis actin gene was used as an internal control. Data are presented as means $\pm S E(n=3)$. * and ** indicate a significant difference from that of WT at $P<0.05$ and $<0.01$, respectively, by Student's t-test.

Two-week-old WT, VC and transgenic plants (lines \#1, \#2, $\# 3$, \#4, \#5 and \#6) were grown in pots under normal condition for 4 weeks. Expression of $V v A A T P$ did not change the growth of transgenic plants since no siggnifcant differences in growth was observed between WT, VC and the transgenic plants under normal conditions. However, the starch content in the leaves of these plants was different. The starch content in VvAATP-expressing plants was increased by $4-81 \%$ compared to that in WT (Figure 3). Further analysis demonstrated that the transgenic plants (\#3 and \#6) had higher starch content than WT, whereas no significant difference was observed between VC and WT plants (Figure 3).

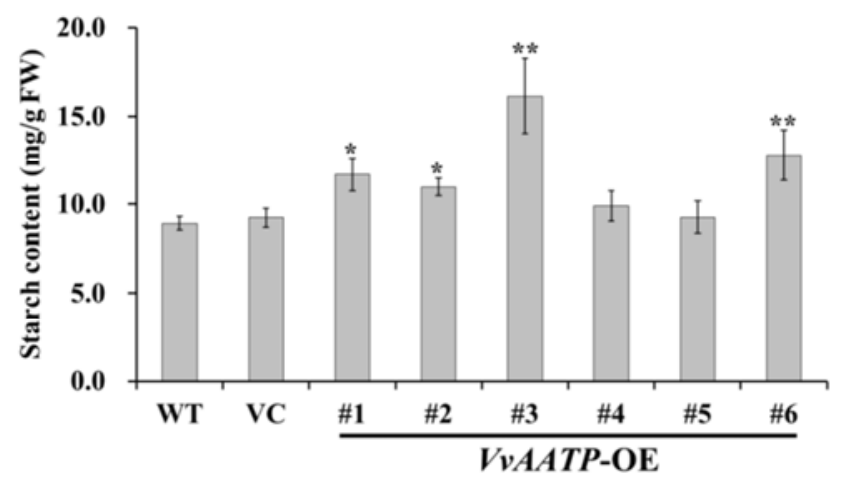

Figure 3. Starch content analyses in the leaves of WT and transgenic plants. Four-week-old WT and transgenic plants grown under normal conditions were used. Data are presented as mean $\pm S E(n=3)$. * and ** indicate a significant difference from that of $W T$ at $P<0.05$ and $<0.01$, respectively, by Student's t-test.

\subsection{Enhanced Starch Biosynthetic Gene Transcription and Enzyme Activity in Arabidopsis Expressing VvAATP}

To dissect how expression of VvAATP increased starch content in transgenic plants, the transcript levels of 13 starch biosynthetic genes in WT, VC and transgenic plants (lines \#3 and \#6) were examined by qRT-PCR (Figure 4). Expression of genes related to starch biosynthesis pathway, such as phosphoglucomutase (AtPGM), AGPase small subunit (AtAGPase-S1 and AtAGPase-S2), AGPase large subunit (AtAGPase-L1 and AtAGPase-L2), granule-bound starch synthase (AtGBSS I and AtGBSS II), soluble starch synthases (AtSSS I, AtSSS II, AtSSS III and AtSSS IV) and starch branching enzyme (AtSBE $I$ and AtSBE $I I)$ was all up-regulated in transgenic plants (Figure 4). These results indicate that $V v A A T P$ might be involved in the regulation of starch biosynthetic processes.

The activity of major enzymes (AGPase, GBSS, SSS and $\mathrm{SBE}$ ) involved in starch biosynthesis was also investigated in the leaves of WT, VC and transgenic plants (lines \#3 and \#6) (Figure 5). The results showed that the activity of these enzymes was significantly enhanced in transgenic plants compared to that in the WT (Figure 5). The increases in enzyme activity in transgenic plants were consistent with the increased transcription levels of their corresponding genes. All these results demonstrate that VVAATP has significant effects on the activities of AGPase, GBSS, SSS and SBE in transgenic Arabidopsis. 


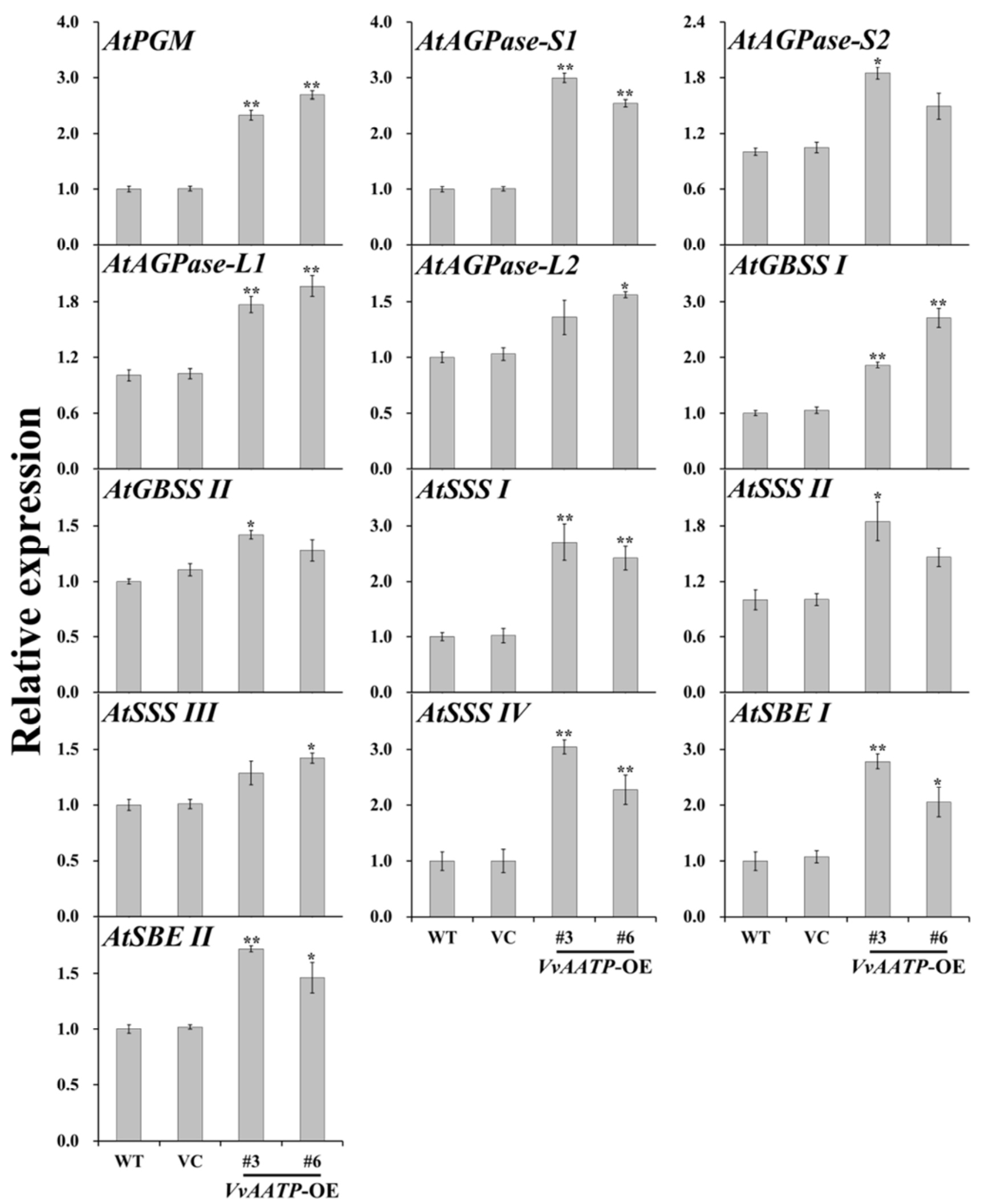

Figure 4. Transcript levels of starch biosynthesis genes in the leaves of WT and transgenic plants. Four-week-old WT and transgenic plants grown under normal conditions were used. The Arabidopsis actin gene was used as an internal control. Results are expressed as relative values with respect to WT, which was set to 1.0. Data are presented as means $\pm S E(n=3)$. * and $* *$ indicate a significant difference from that of WT at $P<0.05$ and $<0.01$, respectively, by Student's $t$-test. 


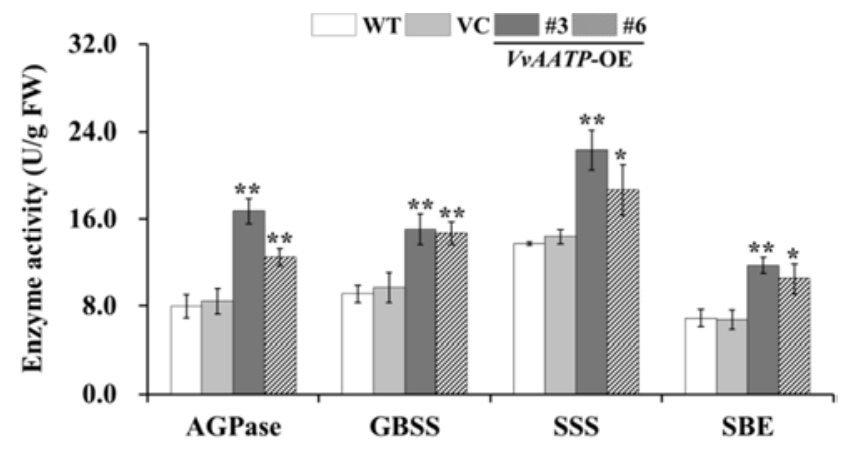

Figure 5. AGPase, GBSS, SSS and SBE enzyme activity assays in the leaves of $W T$ and transgenic plants. Four-week-old WT and transgenic plants grown under normal conditions were used. Data are presented as means $\pm S E(n=3)$. * and ** indicate a significant difference from that of WT at $P<0.05$ and $<$ 0.01 , respectively, by Student's t-test.

\section{Discussion}

Starch is the major dietary source of carbohydrates and the most abundant storage of polysaccharide in higher plants. There a few reports that the AATP gene has been shown to be involved in starch accumulation in plants [17-22]. In this work, the $V v A A T P$ gene was isolated from grapevine. Sequence analysis indicated that $V v A A T P$ protein contained an ADP/ATP carrier protein domain (Figure 1). Constitutive expression of $V v A A T P$ significantly increased starch accumulation in the transgenic Arabidopsis plants (Figure 3).

The carbon precursors and ATP needed for anabolic processes are mainly imported from the cytoplasm in heterotrophic organs [9, 12]. The levels of ATP or ADP-glucose (ADPG) are key targets for genetic manipulation when attempting to accelerate starch biosynthesis in amyloplasts. ATP uptake by amyloplasts is mediated by a plastidic ATP/ADP transporter (AATP) [11]. Constitutive expression of AtAATP1 in potato resulted in the more imported ATP levels from the cytosol into the stroma, which facilitated the synthesis of ADPG, leading to an increase in starch accumulation in tubers [17]. In this study, the starch content in the transgenic plants was significantly increased (Figure 3). Constitutive expression of $V v A A T P$ could also increase ATP import levels into amyloplasts, further activating the pivotal AGPase reaction in starch biosynthesis. Furthermore, we found that the expression of AtAGPase-S1 and AtAGPase-S2, encoding AGPase small subunit, and AtAGPase-L1 and AtAGPase-L2, encoding AGPase large subunit, was up-regulated in transgenic plants (Figure 4). Consistently, AGPase activity was also significantly increased in the $V v A A T P$ expressing Arabidopsis plants (Figure 5). These results showed that ADPG, as a large amount of the ultimate precursor for starch synthesis, could be more accumulated (Figure 6). Meanwhile, the consumption of glucose-1-phosphate (G-1-P) in the AGPase reaction required the accelerated conversion of glucose-6-phosphate (G-6-P) to G-1-P, which was catalyzed by phosphoglucomutase (PGM) [30], and consequently, the transcription level of AtPGM was increased in the transgenic plants (Figure 4). These results suggested that constitutive expression of $V v A A T P$ enhanced the starch biosynthesis due to the increased ATP supply into amyloplasts, which further increased the production of precursors (ADPG and G-1-P) and the expression of starch biosynthesis related genes (Figure 6).

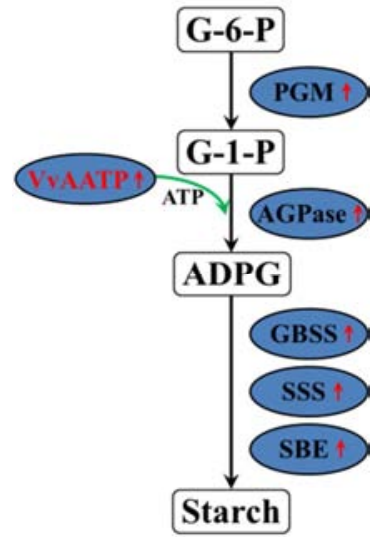

Figure 6. A proposed model of the regulatory network of VvAATP in starch accumulation. Biosynthesis pathway is shown with solid arrows and regulatory interactions with broken arrows. $\uparrow$ indicates up-regulation of the relative enzyme encoding genes.

The higher level of starch content is related to the increased expression of starch biosynthesis genes [5, 31-34]. Starch synthase can be grouped into five types, granule-bound starch synthase (GBSS) and four types of soluble starch synthases (SSS): SSS I, SSS II, SSS III and SSS IV [5, 6, 35]. A large body of evidence has illustrated that up-regulation of these genes can increase starch accumulation in plants [31, 35-38]. In this study, systematic up-regulation of these genes (AtGBSS I, AtGBSS II, AtSSS I, AtSSS II, AtSSS III, AtSSS IV, AtSBE I and $A t S B E I I)$ involved in starch biosynthesis pathway was observed in transgenic plants (Figure 4). Consistent with this phenomenon, the activities of the major enzymes (GBSS, SSS and SBE) involved in starch biosynthesis were also increased in transgenic plants (Figure 5). Therefore, it is thought that constitutive expression of $V v A A T P$ increased the expression of the genes and the activity of the major enzymes involved in starch biosynthesis, leading to the increased starch accumulation in transgenic plants (Figure 6).

\section{Conclusion}

The $V v A A T P$ gene was successfully isolated from grapevine and transformed into Arabidopsis. Constitutive expression of VvAATP significantly increased starch accumulation in the transgenic plants by up-regulating the expression of the related genes and increasing the activities of the major enzymes invovled in starch biosynthesis. Our results suggest that $V v A A T P$ plays a crucial role in starch metabolism, and has potential in the engineering of alternative energy crop plants with improved starch accumulation.

\section{Acknowledgements}

This work was supported by the Natural Science Research 
Project in Colleges of Jiangsu Province of China (17KJB210001), the College Student Practice Innovation Program of Jiangsu Province of China (201711049009H and 201716021YJ) and the Talent Introduction Research Project of Huaiyin Institute of Technology (Z301B16534).

Table 1. Primers used in this study.

\begin{tabular}{|c|c|}
\hline Primer name & Primer sequence $\left(5^{\prime}-3^{\prime}\right)$ \\
\hline \multicolumn{2}{|c|}{ Primers for gene cloning for cDNA } \\
\hline$V \nu A A T P-\mathrm{GC}-\mathrm{F}$ & AATTAGGGAGACATGGAAGCTGTTT \\
\hline VvAATP-GC-R & AATAATGGAAGAAAAATTACTAG \\
\hline \multicolumn{2}{|c|}{ Primers for gene cloning for gDNA } \\
\hline V $A A A T P-\mathrm{GA}-\mathrm{F}$ & GAGACATGGAAGCTGTTTTGCAGAC \\
\hline VvAATP-GA-R & AATTACTAGATGTTGCGAGGAGTT \\
\hline \multicolumn{2}{|c|}{ Primers for constructing expression vector } \\
\hline$V v A A T P-\mathrm{OE}-\mathrm{F}$ & CGGGATCCATGGAAGCTGTTTTGCAGACCAAAG \\
\hline VvAATP-OE-R & TTCGAGCTCCTAGATGTTGCGAGGAGTTGTCTCT \\
\hline \multicolumn{2}{|c|}{ Primers for identifying transformants } \\
\hline hpt II -PCR-F & ACAGCGTCTCCGACCTGATGCA \\
\hline hpt II -PCR-R & AGTCAATGACCGCTGTTATGCG \\
\hline \multicolumn{2}{|c|}{ Primers for real-time quantitative PCR } \\
\hline$V v A A T P-\mathrm{F}$ & TCTGCCCTCAAACCCGAAAT \\
\hline$V v A A T P-\mathrm{R}$ & TCTGGCTCTGCAAACAACGA \\
\hline$A t P G M-\mathrm{F}$ & ATTGCCGTCTCCGTCGTTTA \\
\hline$A t P G M-\mathrm{R}$ & ATGCTATCAGTTCCGGCGAC \\
\hline AtAGPase-S1-F & ACGCACGTATCGGTGACAAT \\
\hline AtAGPase-S1-R & AGTGCCGGTTGGGATTAAGG \\
\hline AtAGPase-S2-F & CCCAAGCGCAGGAATTTACG \\
\hline AtAGPase-S2-R & CGCACCAGGAATGATTTCGC \\
\hline AtAGPase-L1-F & TCTTACCAAGCGAGCAGCAA \\
\hline AtAGPase-L1-R & CGATTGAGGGAAGCCGAG \\
\hline AtAGPase-L2-F & ACTCTTTCGTGTGCACCAGT \\
\hline AtAGPase-L2-R & CAGGTCGACTCCTGTTGGTT \\
\hline AtGBSS I-F & GAACCGTCCCTATTGTGGCA \\
\hline AtGBSS I-R & GCTCTTGTCACAGCCTTTGC \\
\hline AtGBSS II-F & ATCGTTCGATGGATGGAGCC \\
\hline AtGBSS II-R & AACTTCATCTCGTACCGCCC \\
\hline AtSSS I-F & ATTCGAGCCGTTCGTTGGAT \\
\hline AtSSS I-R & AGCAACAATGACCGTCCG \\
\hline AtSSS II-F & GAACCATTCCGGTGGTCCAT \\
\hline AtSSS II-R & TCCGCGCTATCAAATGTCC \\
\hline AtSSS III-F & GGCTCGGCTTGTTCTAACCT \\
\hline AtSSS III-R & TGTGTCAGTCCACATGGCTC \\
\hline AtSSS IV-F & GGAGAATGGTTCTGCGGACA \\
\hline AtSSS IV-R & TCTTGCGCCGAGTAACTTCA \\
\hline AtSBE I-F & AGGCTATTGTTGTCGGCGAA \\
\hline AtSBE I-R & CTGCCACTGCCATGTGTAGA \\
\hline AtSBE II-F & GGGATTAGGCGGTGAAGGTT \\
\hline$A t S B E I I-\mathrm{R}$ & GCCAGGAATCACGCTACCAT \\
\hline Atactin-F & GCACCCTGTTCTTCTTACCGA \\
\hline Atactin-R & AGTAAGGTCACGTCCAGCAAGG \\
\hline
\end{tabular}

\section{References}

[1] Sanz-Barrio, R, Corral-Martinez, P, Ancin, M, et al Overexpression of plastidial thioredoxin $f$ leads to enhanced starch accumulation in tobacco leaves, Plant Biotechnol J. 2013; 11: 618-627.

[2] Smith, AM. Prospects for increasing starch and sucrose yields for bioethanol production, Plant J. 2008; 54: 546-558.

[3] Blennow, A, Jensen, SL, Shaik, SS, et al. Future cereal starch bioengineering cereal ancestors encounter gene technology and designer enzymes, Cereal Chem. 2013; 90: 274-287.
[4] Skryhan, K, Cuesta-Seijo, JA, Nielsen, MM, et al. The role of cysteine residues in redox regulation and protein stability of Arabidopsis thaliana starch synthase 1, PLoS One. 2015; 10: e0136997.

[5] Delvallé, D, Dumez, S, Wattebled, F, et al. Soluble starch synthase I: a major determinant for the synthesis of amylopectin in Arabidopsis thaliana leaves, Plant J. 2005; 43: 398-412.

[6] Fujita, N, Yoshida, M, Asakura, N, et al. Function and characterization of starch synthase I using mutants in rice, Plant Physiol. 2006; 140: 1070-1084.

[7] Jeon, JS, Ryoo, N, Hahn, TR, et al. Starch biosynthesis in cereal endosperm, Plant Physiol Bioch, 2010; 48: 383-392.

[8] Fiore, C, Trézéguet, V, Saux, AL, et al. The mitochondrial ADP/ATP carrier: Structural, physiological and pathological aspects, Biochimie. 1998; 80: 137-150.

[9] Winkler, HH, Neuhaus, HE. Non-mitochondrial ATP transport, Trends Biochem Sci. 1999; 24: 64-68.

[10] Heldt, HW. Adenine nucleotide translocation in spinach chloroplasts, FEBS Lett. 1969; 5: 11-14.

[11] Schünemann, D, Borchert, S, Flügge, UI, et al. ADP/ATP translocator from pea root plastids-Comparison with translocators from spinach chloroplasts and pea leaf mitochondria, Plant Physiol. 1993; 103: 131-137.

[12] Emes, MJ, Neuhaus, HE. Metabolism and transport in non-photosynthetic plastids, J Exp Bot. 1997; 48: 1995-2005.

[13] Möhlmann, T, Tjaden, J, Schwöppe, C, et al. Occurrence of two plastidic ATP/ADP transporters in Arabidopsis thaliana L-Molecular characterization and comparative structural analysis of similar ATP/ADP translocators from plastids and Rickettsia prowazekii, Eur J Biochem. 1998; 252: 353-359.

[14] Linka, N, Hurka, H, Lang, BF, et al. Phylogenetic relationships of non-mitochondrial nucleotide transport proteins in bacteria and eukaryotes, Gene. 2003; 306: 27-35.

[15] Meng K, Chang TJ, Liu X, et al. Cloning and expression pattern of a gene encoding a putative plastidic ATP/ADP transporter from Helianthus tuberosus L., J Integr Plant Biol. 2005; 47: 1123-1132.

[16] Yuen CYL, Leelapon O, Chanvivattana Y, et al. Molecular characterization of two genes encoding plastidic ATP/ADP transport proteins in cassava, Biol Plantarum. 2009; 53: 37-44.

[17] Tjaden J, Möhlmann T, Kampfenkel K, et al. Altered plastidic ATP/ADP-transporter activity influences potato (Solanum tuberosum L.) tuber morphology, yield and composition of tuber starch, Plant J. 1998; 16: 531-540.

[18] Geigenberger P, Stamme C, Tjaden J, et al. Tuber physiology and properties of starch from tubers of transgenic potato plants with altered plastidic adenylate transporter activity, Plant Physiol. 2001; 125: 1667-1678.

[19] Wang, FB, Ye, YX, Niu, Y, et al. A tomato plastidic ATP/ADP transporter gene SlAATP increases starch content in transgenic Arabidopsis, Physiol Mol Biol Plants. 2016; 22: 497-506.

[20] Wang, FB. Fu, LF, Kong, WL, et al. Constitutive expression of StAATP, a potato plastidic ATP/ADP transporter gene, increases starch content in transgenic Arabidopsis, Biotechnol Biotec Eq. 2017; 31(2): 250-258. 
[21] Wang, FB, Chen, XH, Zhang, Fan, et al. A soybean plastidic ATP/ADP transporter gene GmAATP increases starch content in transgenic Arabidopsis, Plant Biotechnol Rep. 2017; 11: 135-146.

[22] Wang, YN, Li, Y, Zhang, H, et al. A plastidic ATP/ADP transporter gene, IbAATP, increases starch and amylose content and alters starch structure in transgenic sweetpotato, J Integr Agr. 2016; 15: 1968-1982.

[23] Lou, XM, Yao, QH, Zhang, Z, et al. Expression of human hepatitis B virus large surface antigen gene in transgenic tomato, Clin Vaccine Immunol. 2007; 14: 464-469.

[24] Zhang, X, Henriques, R, Lin, SS. Agrobacterium-mediated transformation of Arabidopsis thaliana using the floral dip method, Nat Protoc. 2006; 1: 641-646.

[25] Murashige, T, Skoog, F. A revised medium for rapid growth and bioassays with tobacco tissue cultures, Physiol Plant. 1962; 15: 473-497.

[26] Smith, AM, Zeeman, SC. Quantification of starch in plant tissues, Nat Protoc. 2006; 1: 1342-1345.

[27] Li X, Ma H, Huang $\mathrm{H}$, et al. Natural anthocyanins from phytoresources and their chemical researches, Nat Prod Res. 2013; 27 : 456-469.

[28] Schmittgen, TD, Livak, KJ. Analyzing real-time PCR data by the comparative $C_{T}$ method, Nat Protoc. 2008; 3: 1101-1108.

[29] Nakamura, Y, Yuki, K, Park, SY, et al. Carbohydrate metabolism in the developing endosperm of rice grains, Plant Cell Physiol. 1989; 30: 833-839.

[30] Harrison, CJ, Mould, RM, Leech, MJ, et al. The rug3 locus of pea encodes plastidial phosphoglucomutase, Plant Physiol. 2000; 122: 1187-1192.

[31] Jiang, T, Zhai, H, Wang, FB, et al. Cloning and characterization of a carbohydrate metabolism-associated gene IbSnRKI from sweetpotato, Sci Hortic. 2013; 158: 22-32.

[32] Wang, FB, Guo, XT, Qiao, XQ, et al. The maize plastidic thioredoxin F-type gene $Z m \operatorname{Tr} x F$ increases starch accumulation in transgenic Arabidopsis, Sci Hortic. 2016; 210: 205-212.

[33] Wang, FB, Kong, WL, Niu, Y, et al. StTrxF, a potato plastidic thioredoxin F-type protein gene, is involved in starch accumulation in transgenic Arabidopsis thaliana, Biotechnol Biotec Eq. 2017; 31 (3): 486-492.

[34] Wang, FB, Kong, WL, Fu, YR, et al. Constitutive expression of SlTrxF increases starch content in transgenic Arabidopsis, Biol Plant. 2017; 61(3): 494-500.

[35] Szydlowski, N, Ragel, P, Raynaud, S, et al. Starch granule initiation in Arabidopsis requires the presence of either class IV or class III starch synthase, Plant Cell. 2009; 21: 2443-2457.

[36] Burton, RA, Jenner, H, Carrangis, L, et al. Starch granule initiation and growth are altered in barley mutants that lack isoamylase activity, Plant J. 2002; 31: 97-112.

[37] Bustos, R, Fahy, B, Hylton, CM, et al. Starch granule initiation is controlled by a heteromultimeric isoamylase in potato tubers, PNAS. 2004; 101: 2215-2220.

[38] Roldan, I, Wattebled, F, Lucas, MM, et al. The phenotype of soluble starch synthase IV defective mutants of Arabidopsis thaliana suggests a novel function of elongation enzymes in the control of starch granule formation, Plant J. 2007; 49: 492-504. 